\title{
Production of light hypernuclei with light-ion beams and targets
}

\author{
Y. L. Sun, ${ }^{1, *}$ A. S. Botvina, ${ }^{2,3}$ A. Obertelli,,${ }^{1,4}$ A. Corsi, ${ }^{1}$ and M. Bleicher ${ }^{2}$ \\ ${ }^{1}$ Département de Physique Nucléaire, IRFU, CEA, Université Paris-Saclay, F-91191 Gif-sur-Yvette, France \\ ${ }^{2}$ Frankfurt Institute for Advanced Studies and ITP J. W. Goethe University, D-60438 Frankfurt am Main, Germany \\ ${ }^{3}$ Institute for Nuclear Research, Russian Academy of Sciences, 117312 Moscow, Russia \\ ${ }^{4}$ Institut für Kernphysik, Technische Universität Darmstadt, D-64289 Darmstadt, Germany
}

(Received 23 November 2017; published 7 August 2018)

\begin{abstract}
Ion-ion collisions at relativistic energies have been shown recently to be a promising technique for the production of hypernuclei. In this article, we further investigate the production of light $\Lambda$ hypernuclei by use of a hybrid dynamical model: cascade coalescence followed by Fermi breakup. The predictions are then compared with the available experimental data. The dependence of the production cross section on the beam energy, beam mass number, as well as different projectile-target combinations is investigated. In particular, we evaluate the yields and signal-over-background ratio in the invariant-mass spectrum for carbon projectiles impinging on hydrogen and carbon targets and various coincidence conditions in the experiment using the theoretical calculation as an input. It is found that, comparing with the carbon target, the hydrogen target also leads to sizable hypernuclear yields, even for exotic species, and the hydrogen target could improve significantly the signal-over-background ratio in some hypernuclear invariant mass studies.
\end{abstract}

DOI: 10.1103/PhysRevC.98.024903

\section{INTRODUCTION}

Hyperons $(\Lambda, \Sigma, \Xi, \Omega)$ are baryons containing at least one strange quark, unlike nucleons (proton or neutron) which are only composed of $u$ and $d$ valence quarks. The free $\Lambda$ particle, the lightest hyperon, can only decay to a pion-nucleon system through the weak interaction, since the strong interaction conserves strangeness. Interestingly, it was discovered that hyperons can form bound systems with nucleons and create short-lived hypernuclei [1]. The investigation of hypernuclei provides a practical method to study the fundamental hyperonnucleon $(Y N)$ and hyperon-hyperon $(Y Y)$ interactions in nuclear matter at low energies. Indeed, the very short lifetime of $\Lambda$ (263 ps [2]) makes it technically extremely difficult to use lambda particles directly as projectiles for scattering or capture experiments.

Starting from the end of 1960s, the emulsion technique with cosmic rays and the missing mass technique using ${ }^{A} Z\left(K^{-}, \pi^{-}\right)_{\Lambda}^{A} Z$ and ${ }^{A} Z\left(\pi^{+}, K^{+}\right)_{\Lambda}^{A} Z$ reactions have been widely used to produce hypernuclei in the laboratory [3]. As the lambda particle can be distinguished from nucleons by the strangeness quantum number and is not limited by the Pauli exclusion principle [4], adding a lambda particle to a nucleus tends to increase the binding energies of the whole system. The measured binding energies thus give information on the $Y N$ interaction. Moreover, the glue-like role of the hyperon is expected to change the nuclear deformation $[5,6]$, to lead to new excitation modes [6], and to shift the neutron and proton drip lines from their normal limits $[7,8]$. They have been discovered to bind loosely unbound nuclei at the drip line,

*yelei.sun@cea.fr such as ${ }^{5} \mathrm{H}$ and ${ }^{7} \mathrm{He}$ (via ${ }_{\Lambda}^{6} \mathrm{H} \mathrm{[9]}$ and ${ }_{\Lambda}^{8} \mathrm{He}$ [10], respectively). Hyperons are also expected to be of major interest for nuclear structure: due to the absence of Pauli blocking with nucleons, they constitute a unique opportunity to probe the inner densities in nuclei. Furthermore, in the field of astrophysics, hyperons are predicted to exist inside neutron stars at densities exceeding 2-3 $\rho_{0}$, where $\rho_{0}=0.16$ baryon $/ \mathrm{fm}^{3}$, which is the nuclear saturation density. However, depending on the detailed properties of the $Y Y$ interaction and $Y N N$ three-body interaction, the presence of hyperons in neutron stars can either soften or stiffen the high-density equation of state (EOS), resulting in large uncertainty in the prediction of the maximum mass of neutron stars $[11,12]$. The study of neutron-rich hypernuclei may provide relevant information to solve this hyperon puzzle and help us to achieve a better description on the EOS of high density nuclear matter and the evolution of compact stars.

Although many theoretical works predict the existence of neutron-rich or proton-rich hypernuclei, up to now most of the produced hypernuclei are limited to systems close to the stability line. Very recently, two distinguished results were obtained using the double charge exchange reaction ${ }^{6} \mathrm{Li}\left(K_{\text {stop }}^{-}, \pi^{+}\right)_{\Lambda}^{6} \mathrm{H}$ [9] and the electroproduction reaction ${ }^{7} \mathrm{Li}\left(e, e^{\prime} K^{+}\right){ }_{\Lambda}^{7} \mathrm{He}$ [13], with very small cross sections $(\approx 10 \mathrm{nb} / \mathrm{sr})$. The production of heavier exotic hypernuclei with large isospin asymmetry using the techniques mentioned above is not feasible. Reactions with ion beams at relativistic energies provide an alternative approach to overcome this bottleneck. Searching for hypernuclei using ion beams can be traced back to the 1970s at Berkeley [14] and later in Dubna [15], although at that time the produced hypernuclei were only signaled by hardware trigger selection without detailed particle identification. With the improvement of the experimental setup, ${ }_{\Lambda}^{3} \mathrm{H}$ and ${ }_{\Lambda}^{4} \mathrm{H}$ were successfully identified in central collisions by impinging $11.5 \mathrm{GeV} / c$ 
platinum beams on a Au target [16]. It is generally interesting that in ultrarelativistic ion collisions some light hypernuclei, e.g., hypertritons $\left({ }_{\Lambda}^{3} \mathrm{H}\right)$ and the corresponding antiparticles $\left(\frac{3}{\bar{H}}\right)$, can be produced in the mid-rapidity region, as was measured by the STAR [17] and ALICE [18] Collaborations. The mechanisms for producing such species at very high energies and temperatures are still under intensive discussion. The coalescence mechanism is one of the candidates [19] and it can also be applied to explain the formation of hyper-species outside of the mid-rapidity region [20], where the production of larger hypernuclei is possible.

More recently, the known hypernuclei ${ }_{\Lambda}^{3} \mathrm{H}$ and ${ }_{\Lambda}^{4} \mathrm{H}$, with their lifetimes, were measured in the projectile rapidity region by the HypHI Collaboration via fragmentation of a $2 \mathrm{~A} \mathrm{GeV}$ ${ }^{6} \mathrm{Li}$ beam impinging on a carbon target [21,22]. The experiment successfully demonstrates the feasibility of producing hypernuclei in peripheral collisions. Note that in this technique large fragments of projectile and target nuclei do not interact with each other intensively and form spectator residues, which might capture the hyperons if momentum matching allows. Due to the large Lorentz boost, the production and decay vertices can be well separated by tens of centimeters, making it possible to identify effectively the production and decay of hypernuclei independently. In addition, a possible existence of ${ }_{\Lambda}^{3} n$ was suggested, which might come from the disintegration of heavier projectile-like hyperfragments [22], implying a new mechanism of producing exotic hypernuclei. In the near future, the FAIR [23,24] facility in GSI and HIAF [25] facility in China will provide high energy and high intensity ion beams, providing good opportunities to study projectile-like hypernuclei. Proton-rich and neutron-rich hypernuclei are foreseen to be produced efficiently using various secondary beams $[8,26]$.

In the present article, we focus on the production of hypernuclei in peripheral ion collisions. The production of hypernuclei is investigated in the whole rapidity region by considering the cascade coalescence as well as the projectile and target decay processes. We first give a description of our model in Sec. II and then compare the calculation results with few existing data in Sec. III. We then present in Sec. IV new calculations to investigate the energy dependence, the projectile, and target dependence to produce light $\Lambda$ hypernuclei. Finally, in Sec. V, we comment on the signal-over-background ratio as a function of target-projectile combinations and particle coincidences in possible future experiments.

\section{MODEL DESCRIPTION}

In the present work, we adopt the approach of using the dynamical cascade followed by statistical deexcitation to describe the formation of hypernuclei in ion-ion collisions. This approach has been shown to be very successful for the description of normal fragment production; see Refs. [27,28]. Recently, this approach was extended to describe the formation of hypernuclei $[8,29,30]$. In hadron and ion high-energy collisions, the main production sources of hyperons are nucleonnucleon collisions, e.g., $p+p \rightarrow p+\Lambda+K^{+}$(threshold $E_{\mathrm{lab}} \geqslant 1.58 \mathrm{GeV}$ ) and also secondary meson-nucleon collisions, e.g., $\pi^{+}+n \rightarrow \Lambda+K^{+}$(threshold $E_{\text {lab }} \geqslant 0.76 \mathrm{GeV}$ ). At energies lower than $2 \mathrm{GeV} /$ nucleon, these elementary colli- sions are reliably described by using the available experimental data and phenomenological parametrizations. Experimental cross sections, or calculated cross sections if data are not available, are used to calculate the angular and energy distributions of outgoing channels. Generally, at higher energies, the formation of hyperons is usually estimated by hadronization models, such as the quark gluon string model (QGSM) [31], PYTHIA [32] or the Lund-Fritiof string model [33]. Subhadronic degrees of freedom, such as quarks and strings, are taken into account phenomenologically. Afterwards, the evolution of hadrons (mesons + baryons) in space and time may be described with different transport models, e.g., intranuclear cascade models (like DCM [31,34] and INC [35]), UrQMD [36,37], GiBUU [38], and HSD [39], by solving the relativistic Boltzmann transport equations. All these models have been shown to be successful in the description of hyperon production $[20,29,40]$.

During the transportation, the capture of lambda particles and other particles by the neighboring nuclear fragments is determined either by the coalescence criterion [20,41,42], i.e., hypernuclei are formed when hyperons are close to nucleons in both the spatial and momentum space, or by the potential criterion [29], i.e., hypernuclei are formed when the kinetic energies of hyperons in the residue-at-rest frame are smaller than the attractive potential of the residues. Since the primarily produced $\Lambda$ particles have usually large momentum mismatch with the fragments and thus can hardly be captured, rescattering and secondary interactions are important for the capture process [43]. A comparison of the capture parameters and hyperresidues obtained in DCM and UrQMD can be found in Refs. [29,30], where qualitative agreement was reported.

After the nonequilibrium and absorption stages $(\tau \approx$ 50-100 fm/c), as expected for normal residual nuclei $[44,45]$, the formed primary hyperresidues are usually in high excited states. They may deexcite with production of cold normal nuclei and hypernuclei [46], which could take time ranging from $10^{2}$ to $10^{4} \mathrm{fm} / c[28,29]$. Various deexcitation processes can take place depending on the excitation energy and the mass number of the hyperresidues. For light excited hyperresidues $(A \leqslant 16)$, the Fermi-breakup model can be applied $[8,47]$. While for larger species, the evaporation-fission model $\left(E^{*}<\right.$ 2-3 MeV/nucleon) [48], or multifragmentation model [46] at higher excitation energy can be used.

In the present work we adopted the Dubna intranuclear cascade model (DCM) $[8,34]$ for description of collisions with relatively light nuclei $(A \leqslant 16)$. The details of DCM can be found in many references; see [8,29-31,34]. DCM includes up to 70 baryonic species and includes all the conservation laws in elementary binary interactions of hadrons related to the scattering and production of new particles. For elementary hadron collisions at relatively low energy $\left(E_{\text {lab }}<1-3 \mathrm{GeV} /\right.$ nucleon, where $E_{\text {lab }}$ is the laboratory energy of the colliding nuclei) the model uses quite reliable approximations for the reaction channels, supported by the analysis of a large amount of available experimental data [30]. In addition, at higher energies ( $E_{\text {lab }}>4.5 \mathrm{GeV} /$ nucleon), the QGSM [29,31] is involved in the smooth transition between these two limits [30]. There are many comparisons of DCM with experimental data on particle production. The latest analyses of the lambda hyperon 
yields in DCM can be found in Refs. [29,30], confirming the reliability of the DCM model for description of strangeness production. As described before, hypernuclei are formed when the produced hyperons are captured by neighboring nucleons or residual fragments.

For the capture by residues, we took the potential criterion. The hyperon potential in cold nuclear matter at saturation density was taken as $30 \mathrm{MeV}$, and a correction on the density variation was applied [29]. The capture takes place during the cascade process until a few tens of $\mathrm{fm} / c$ when the colliding light nuclei pass through each other. After the cascade and capture stages, as expected for normal residues [28], we assume the hyperresidues reach statistical equilibrium. The deexcitation of the hot hyperresidues was described by the Fermi-breakup model [8,47]. In this model we have considered the decay channels of hot primary hyperresidues into all existing hypernuclei $(A \leqslant 16):{ }_{\Lambda}^{3,4} \mathrm{H},{ }_{\Lambda}^{4,5,6} \mathrm{He},{ }_{\Lambda}^{6,7,8,9} \mathrm{Li},{ }_{\Lambda}^{7,8,9,10} \mathrm{Be}$, ${ }_{\Lambda}^{10,11,12} \mathrm{~B},{ }_{\Lambda}^{12,13,14} \mathrm{C},{ }_{\Lambda}^{14,15} \mathrm{~N},{ }_{\Lambda}^{16} \mathrm{O}$. Some neutron-rich hypernuclei, ${ }^{\Lambda}$ such as ${ }_{\Lambda}^{3} n,{ }_{\Lambda}^{6} \mathrm{H}$, and ${ }_{\Lambda}^{8} \mathrm{He}$, were included so that we could examine the production yields of exotic species. Also, for complete analysis, exotic ${ }_{\Lambda}^{2} \mathrm{H}$ and ${ }_{\Lambda}^{2} n$ hypersystems were considered with masses equal to the sum of the masses of constituents. If there are no such bound states in reality, these species should be taken as unbound particles.

After the first dynamical stage, besides the hyper"spectator" residues, we obtain different particles over all of the rapidity region which can also form clusters as a result of the final state interaction. The coalescence model developed in Refs. $[20,34]$ was used by us to describe it. For this case we investigate the proximity of produced baryons in the phase space on an event-by-event basis. The coalescence parameters have been taken from the previous works [34,42] and they describe very good experimental data on the yields of normal light nuclei at intermediate energies. This is an additional source of hypernuclei which is calculated in our work too.

In Fig. 1, we give an example for ${ }^{12} \mathrm{C}+{ }^{12} \mathrm{C}$ collision at $2 A \mathrm{GeV}$. The rapidities of $\Lambda$, projectile-like, and target-like hypernuclei in the center-of-mass frame are shown in different colors. The yield of each component was normalized by the total number of the inelastic collisions. From Fig. 1, we can see that the produced lambda hyperons have a very broad rapidity distribution. The broadening is mainly due to the $\Lambda N$ elastic scattering whose typical cross section is around $30 \mathrm{mb}$ [29]. Projectile-like and target-like hypernuclei can be formed in the overlap rapidity region between the residues and the lambda hyperons.

In the calculation of deexcitation processes, the excitation energy of the primary hypernuclei is determined afterward. We use the approximation described in Ref. [49], and adopt the following relation between the residue mass and the average excitation energy:

$$
A / A_{0}=1-a_{1}\left(E_{x} / A\right)-a_{2}\left(E_{x} / A\right)^{2}
$$

where $a_{1}=0.08983 \mathrm{MeV}^{-1}, a_{2}=0.007728 \mathrm{MeV}^{-2}, A$ is the mass number of the hyperresidue, and $A_{0}$ is the mass number of the projectile or the target. This correlation is consistent with the result obtained in the DCM calculations for light collision system $\left({ }^{12} \mathrm{C}+{ }^{12} \mathrm{C}\right.$ at $\left.5 \mathrm{~A} \mathrm{GeV}\right)[30]$, which is demonstrated by

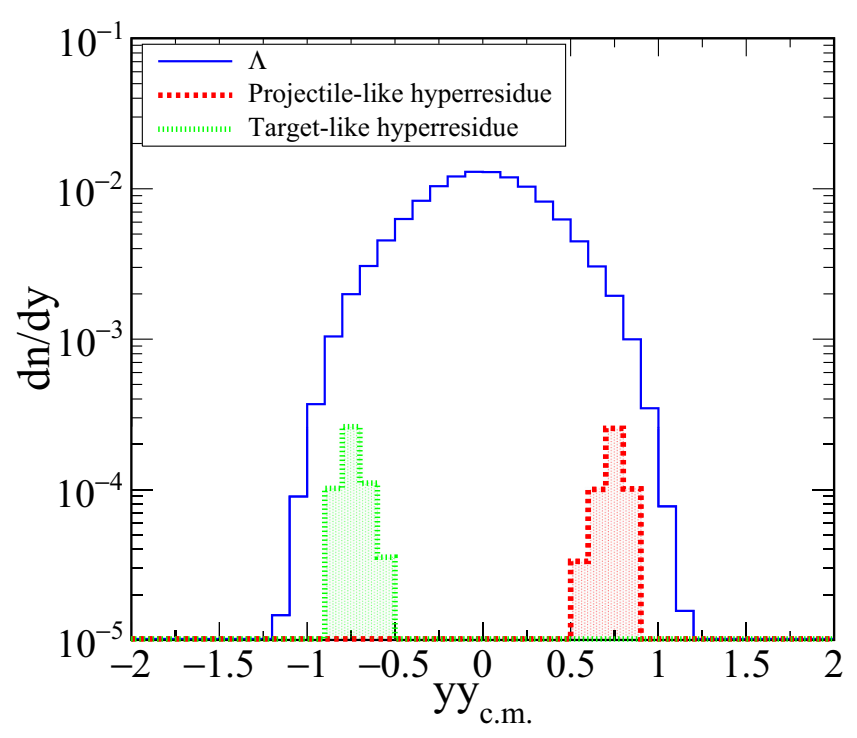

FIG. 1. Rapidity distributions of $\Lambda$ hyperons and hyperresidues in the center-of-mass frame as calculated in the DCM model for ${ }^{12} \mathrm{C}(2 \mathrm{~A} \mathrm{GeV})+{ }^{12} \mathrm{C}$ collisions. The solid blue line gives the rapidity of $\Lambda$ hyperons. Red and Green histograms show the rapidity of projectile-like and target-like hyperresidues, respectively.

the black symbols in Fig. 2. To further evaluate the sensitivity of the hypernuclear yields to the excitation energy of the primary hyperresidues, we explore two other excitation-energy distributions. $E_{x}$ has uniform distribution from 0 to $1 A \mathrm{MeV}$ and from $1 \mathrm{~A}$ to $5 \mathrm{~A} \mathrm{MeV}$, corresponding to red and blue

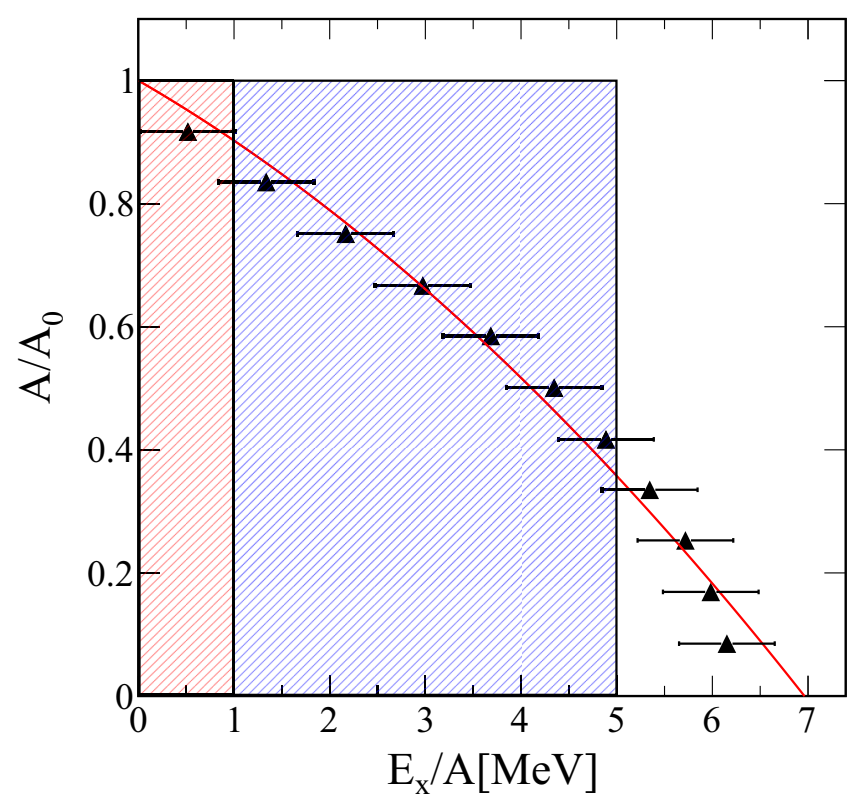

FIG. 2. Excitation-energy distributions used in the present theoretical calculation. The red curve shows the parametrized excitationenergy distribution of Eq. (1). The red and blue shadow areas show the other two uniform excitation-energy distributions adopted in the calculation. The black points are taken from Ref. [30], which are the excitation energies extracted using DCM. 


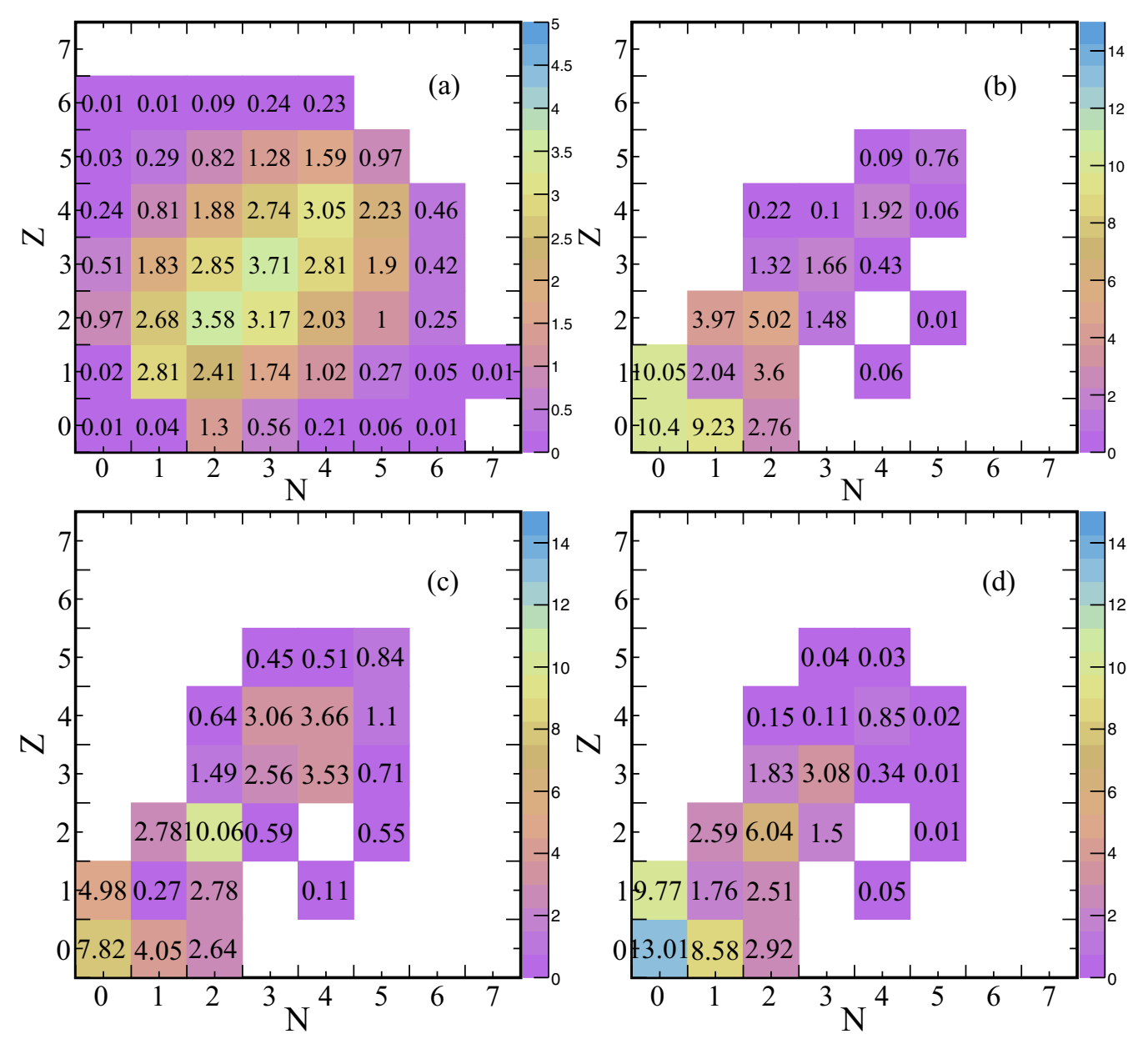

FIG. 3. Hypernuclear production cross sections for ${ }^{12} \mathrm{C}+{ }^{12} \mathrm{C}$ at $2 A \mathrm{GeV}$ : (a) without Fermi breakup, (b) with Fermi breakup and parametrized $E_{x}$ distribution, (c) with Fermi breakup and $E_{x}=0-1 A \mathrm{MeV}$ uniform distribution, and (d) with Fermi breakup and $E_{x}=1 A-5 A$ $\mathrm{MeV}$ uniform distribution. The units of the cross sections are $\mu \mathrm{b}$. Only projectile-like species are considered.

shadow areas in Fig. 2. The production cross sections of hyperfragments from ${ }^{12} \mathrm{C}+{ }^{12} \mathrm{C}$ at $2 \mathrm{GeV} /$ nucleon is shown in Fig. 3. Panel (a) shows the production cross sections for all projectile-like residues after the cascade but before deexcitation while panel (b) shows the production cross sections after deexcitation following the parametrization of Eq. (1). Panels (c) and (d) show the production cross sections after the deexcitation with excitation energy uniformly distributed from 0 to $1 A \mathrm{MeV}$ and from $1 A$ to $5 A \mathrm{MeV}$. As shown in Fig. 3(a), without deexcitation, just after the cascade-coalescence stage, many hot primary hyperresidues could be produced. However, after considering the deexcitation, as shown in Figs. 3(b)$3(d)$, most of the remaining cold hypernuclei locate close to the $\beta$-stability line with yields around several microbarns. Neutron-rich or proton-rich hypernuclei could also survive with relatively smaller probability. Cross sections of ${ }_{\Lambda}^{6} \mathrm{H}$ and ${ }_{\Lambda}^{8} \mathrm{He}$ are on the order of few to several tens of nanobarns. Note that ${ }_{\Lambda}^{5} \mathrm{H}$ and ${ }_{\Lambda}^{7} \mathrm{He}$ were not considered as bound systems at the breakup stage, which results in empty holes in the $Z-N$ plane. The hypernuclear yields in the present work are smaller than those in Ref. [26], and the form of $Z-N$ plane population is different. The differences are due to that in Ref. [26] the geometrical cross sections instead of the inelastic cross sections were wrongly taken to estimate the hypernuclear yields, and breakup of the hot primary hyperresidues was not considered [50]. Actually, the production cross sections are quite sensitive to the adopted excitation energies, since the breakup probability is proportional to $\left(E_{x}-U\right)^{3 n / 2-5 / 2}$, where $U$ is the Coulomb barrier and $n$ is the number of cold residues after breakup [47]. Especially for heavier hyperfragments, the resulting cross sections could be more than one order of magnitude of difference. If no special indication is given, the following calculations are performed with the parametrization of Eq. (1) for the excitation-energy function.

\section{BENCHMARK WITH EXISTING DATA}

Until now, the experimental cross sections of ion induced hypernuclear production have been very scarce. Two available data were measured at Dubna [15] and at GSI by the HypHI Collaboration [51]. Both measurements use ion beams impinging on a carbon target and focused on the formed hypernuclei around the beam rapidity region. The theoretical projectile-like hypernuclear yields and the experimental data are summarized in Table I. 
TABLE I. The calculated cross sections are compared with the Dubna and HypHI data. Three excitation-energy distributions are considered in the calculation: (I) parametrized $E_{x}$ distribution; (II) and (III) $E_{x}$ uniformly distributed from 0 to $1 A \mathrm{MeV}$ and $1 A$ to $5 A \mathrm{MeV}$, considered as test excitation-energy distributions without theoretical foundations. The unit of the cross sections are given in $\mu$ b. Only projectile-like hypernuclei are considered.

\begin{tabular}{|c|c|c|c|c|}
\hline Beam & Energy (GeV/nucleon) & & ${ }_{\Lambda}^{3} \mathrm{H}$ & ${ }_{\Lambda}^{4} \mathrm{H}$ \\
\hline${ }^{3} \mathrm{He}$ & 5.14 & $\begin{array}{c}\text { (I) } \\
\text { (II) } \\
\text { (III) } \\
\text { Dubna [15] }\end{array}$ & $\begin{array}{c}0.63 \\
0.05 \\
<0.01 \\
0.05_{-0.02}^{+0.05}\end{array}$ & \\
\hline${ }^{4} \mathrm{He}$ & 3.7 & $\begin{array}{c}\text { (I) } \\
\text { (II) } \\
\text { (III) } \\
\text { Dubna [15] }\end{array}$ & $\begin{array}{c}<0.01 \\
0.24 \\
0.04 \\
<0.1\end{array}$ & $\begin{array}{c}0.19 \\
0.12 \\
<0.01 \\
0.4_{-0.2}^{+0.4}\end{array}$ \\
\hline${ }^{6} \mathrm{Li}$ & 3.7 & $\begin{array}{c}\text { (I) } \\
\text { (II) } \\
\text { (III) } \\
\text { Dubna [15] }\end{array}$ & $\begin{array}{c}1.15 \\
0.29 \\
0.84 \\
0.2_{-0.15}^{+0.3}\end{array}$ & $\begin{array}{c}0.27 \\
2.31 \\
0.33 \\
0.3_{-0.15}^{+0.3}\end{array}$ \\
\hline${ }^{6} \mathrm{Li}$ & 2.0 & $\begin{array}{c}\text { (I) } \\
\text { (II) } \\
\text { (III) } \\
\text { HypHI [51] }\end{array}$ & $\begin{array}{c}0.2 \\
0.03 \\
0.13 \\
3.9 \pm 1.4\end{array}$ & $\begin{array}{c}0.02 \\
0.43 \\
0.04 \\
3.1 \pm 1.0\end{array}$ \\
\hline
\end{tabular}

Here, we note that in the cases of $\left({ }^{3} \mathrm{He},{ }_{\Lambda}^{3} \mathrm{H}\right)$ and $\left({ }^{4} \mathrm{He},{ }_{\Lambda}^{4} \mathrm{H}\right)$, one proton in the projectile is substituted by a lambda particle. In the calculation using the parametrized excitation-energy distribution, the yields of ${ }_{\Lambda}^{3} \mathrm{H}$ and ${ }_{\Lambda}^{4} \mathrm{H}$ are not reduced by the decay processes, since the formed hyperresidues have the same mass number as the projectiles, and the excitation energies deduced from Eq. (1) are always zero. The model with such excitation-energy distribution thus is useful only for reactions of deep disintegrations of large projectiles. Instead, the other two excitation-energy distributions $\left(0<E_{x} / A<1 \mathrm{MeV}\right.$ or $1<E_{x} / A<5 \mathrm{MeV}$ ) reach a better agreement with the Dubna data. In the case of ${ }^{6} \mathrm{Li}$ projectile at $3.7 \mathrm{GeV} /$ nucleon, the parametrized excitation-energy distribution reproduces the yield of ${ }_{\Lambda}^{4} \mathrm{H}$, but overestimates the yield of ${ }_{\Lambda}^{3} \mathrm{H}$, while a smaller excitation energy $\left(0<E_{x} / A<1 \mathrm{MeV}\right)$ gives a better result. The excitation-energy distribution thus seems to have a more complicated dependence on the mass ratio $\left(A / A_{0}\right)$ than that in Eq. (1) for lightest projectiles, which is difficult to benchmark at this stage with a limited data set. Nevertheless, we conclude that a satisfactory agreement is found with the Dubna data. On the other hand, we found that the calculated yields of ${ }_{\Lambda}^{3} \mathrm{H}$ and ${ }_{\Lambda}^{4} \mathrm{H}$ in ${ }^{6} \mathrm{Li}+{ }^{12} \mathrm{C}$ collisions at $2 \mathrm{GeV} /$ nucleon are more than one order of magnitude smaller than the HypHI data. Surprisingly, we found that none of the excitation distributions could result in the same order of magnitude of cross sections as the HypHI experiment. To investigate the reasons, we further compare the rapidity and transverse momentum distributions with the experimental data. Note that here we can do direct comparison since the experimental rapidity and transverse momentum distributions have already been corrected by the experimental acceptance and reconstruction efficiency [51].
The results are shown in Fig. 4. In panels (a) and (b), the distributions are plotted as a function of the particles' rapidity in the center-of-mass reference frame of the individual $N N$ collisions, which is scaled to the rapidity of this reference frame: $y_{0}=\left(y_{\mathrm{lab}}-y_{\mathrm{cm}}\right) / y_{\mathrm{cm}}$, where $y_{\mathrm{cm}}$ denotes the rapidity of the center-of-mass reference frame of the individual $N N$ collisions. ${ }^{1}$ The projectile-like, target-like, and cascadecoalescence contributions of ${ }_{\Lambda}^{3} \mathrm{H}$ and ${ }_{\Lambda}^{4} \mathrm{H}$ are shown in different colors. The distributions are normalized by the total number of the inelastic collisions. Experimental data from Ref. [51] are renormalized by the rapidity bin size (See Fig. 3 of Ref. [51]). The $y$ axis therefore stands for the multiplicity per inelastic collision per unit of rapidity. Consistent with the cross sections, the amplitudes of the rapidity distributions of the projectile-like ${ }_{\Lambda}^{3} \mathrm{H}$ and ${ }_{\Lambda}^{4} \mathrm{H}$ are much smaller than the data. We also found that there exists some shift between the theoretical and experimental rapidity distributions, which is due to the dissipative processes in the cascade calculation. In addition, we found that ${ }_{\Lambda}^{3} \mathrm{H}$ coming from the coalescence of the cascade particles can also locate around the beam rapidity region. With these contributions, the theoretical yield of ${ }_{\Lambda}^{3} \mathrm{H}$ still underestimate the HypHI data by more than one order of magnitude. In the case of ${ }_{\Lambda}^{4} \mathrm{H}$, there is no contribution from cascade-coalescence particles in the beam rapidity region. To estimate the maximum yields of ${ }_{\Lambda}^{3} \mathrm{H}$ and ${ }_{\Lambda}^{4} \mathrm{H}$, we also give the rapidity distributions of mother hypernuclei for ${ }_{\Lambda}^{3} \mathrm{H}$ and ${ }_{\Lambda}^{4} \mathrm{H}$, which are shown by the red dashed lines. The mother hypernuclei represent all the possible hot primary hyperresidues which could decay to ${ }_{\Lambda}^{3} \mathrm{H}$ or ${ }_{\Lambda}^{4} \mathrm{H}$. Still, we found that even in this case the estimated maximum yields are smaller than the experimental data. Nevertheless, we should mention that our calculated total yield of $\Lambda$ hyperons is around $4.5 \mathrm{mb}$, which is even larger than the HypHI acceptance-corrected result of $1.7 \pm 0.8 \mathrm{mb}$ [51]. If the reason for the low hypernuclear yields in the calculation is a too small probability for capture of hyperons by residues in our model, then one can consider our calculations as lower limit predictions. This makes future hypernuclear experiments even more promising, since it allows for knowledge of the hyperon capture potential in excited nuclei, which, in principle, could be larger than in cold nuclei. In panels (c) and (d), we give the transverse momentum distributions of ${ }_{\Lambda}^{3} \mathrm{H}$ and ${ }_{\Lambda}^{4} \mathrm{H}$. The experimental data from the HypHI experiment are shown by black points. The spectra in red and blue represent the contributions of projectile-like and target-like hypernuclear species, respectively. In this plot, the theoretical distributions are normalized by the data. From the transverse momentum distributions, we can clearly see that the observed ${ }_{\Lambda}^{3} \mathrm{H}$ and ${ }_{\Lambda}^{4} \mathrm{H}$ in the HypHI experiment are mainly the projectile-like component, as the cascade-coalescence hypernuclei have a much broader transverse momentum distribution.

We found that recently there was another attempt to describe the HypHI data, which was published as a conference proceeding [52]. The authors used the IQMD dynam-

\footnotetext{
${ }^{1}$ For the collision of ${ }^{6} \mathrm{Li}+{ }^{12} \mathrm{C}$ at $2 A \mathrm{GeV}, y_{\mathrm{cm}}$ is about 0.91 . This $y_{\mathrm{cm}}$ definition coincides with the rapidity in the equal velocity system for asymmetric nuclei collisions.
} 

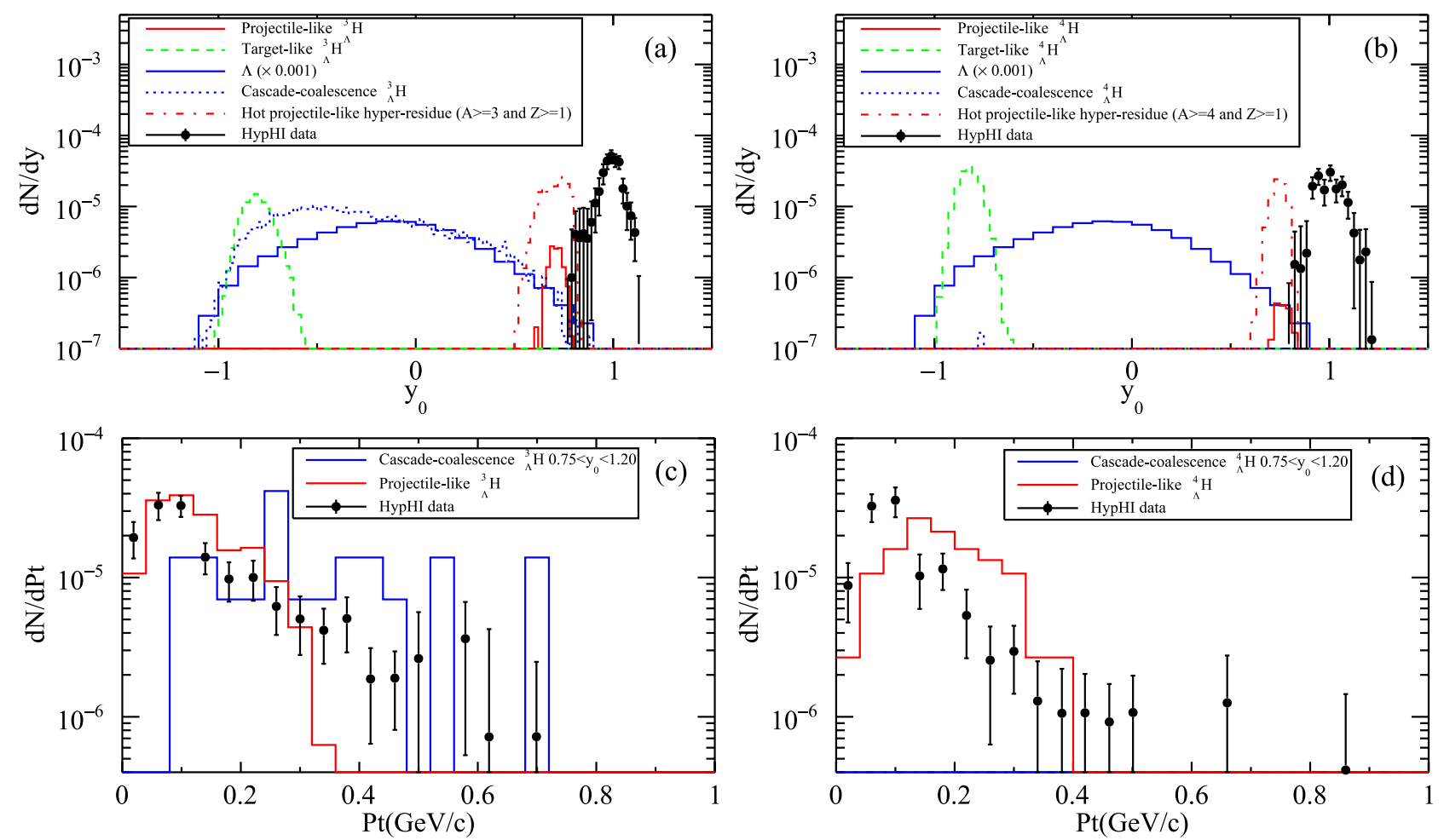

FIG. 4. Rapidity and transverse momentum distributions are compared with the experimental data of HypHI. $y_{0}$ denotes the rapidity in the center-of-mass frame of the individual $N N$ collisions scaled to the rapidity of this reference frame and $P_{t}$ is the transverse momentum. Panels (a) and (b) show the rapidity results related to ${ }_{\Lambda}^{3} \mathrm{H}$ and ${ }_{\Lambda}^{4} \mathrm{H}$. The projectile-like, target-like, and cascade-coalescence hypernuclei are shown in different colors. The experimental data from the HypHI experiment [51] are shown as black points. Data are renormalized by dividing by the rapidity bin size of 0.02 for ${ }_{\Lambda}^{3} \mathrm{H}$ or 0.03 for ${ }_{\Lambda}^{4} \mathrm{H}$ (see Fig. 3 of Ref. [51]). The rapidity distributions are normalized by the total number of the inelastic collisions. $d N / d y$ is therefore the multiplicity per inelastic collision per unit of rapidity. Panels (c) and (d) show the transverse momentum of ${ }_{\Lambda}^{3} \mathrm{H}$ and ${ }_{\Lambda}^{4} \mathrm{H}$. The projectile-like and cascade-coalescence contributions in the forward rapidity region are shown in different colors. Data are renormalized by dividing by the momentum bin size of $40 \mathrm{MeV} / c$ (see Fig. 3 of Ref. [51]). The theoretical transverse momentum distributions are normalized by the data.

ical description with a coalescence-like procedure (FRIGA semiclassical method) for formation of clusters including hypernuclei. Within this method the hyperclusters are considered as cold nuclei without subsequent deexcitation. Therefore, it can be applied only for the lightest species of hypernuclei. The calculated distributions of ${ }_{\Lambda}^{3} \mathrm{H}$ and ${ }_{\Lambda}^{4} \mathrm{H}$ over the full range of rapidity are presented. Note that the experimental data presented in Fig. 3 of Ref. [52] were not normalized by the rapidity bin size of 0.02 as presented in Fig. 3 of Ref. [51], and this gives an impression of low experimental yields. With correct normalization, the yields in Ref. [52] in the projectile-like rapidity region are essentially smaller than the experimental ones, similar to our results. Still there are discrepancies of our calculations with the IQMD rapidity distributions of hypernuclei, since they predict a nearly symmetric form with respect to the central rapidity. In our case, a heavier target (carbon) leads to enhanced production of hypermatter in the target rapidity region. Our difference between projectileand target-like rapidity of all fragments is smaller because of the kinetic energy loss during the particle production. Also relative to the experimental transverse momentum distributions of HypHI, there is about $0.1 \mathrm{GeV} / c$ shift from the calculations in Ref. [52]. While in our case the overall shape of the experimental transverse momentum distributions can be well described by the projectile-like component. No shift was found in our calculation results in the case of ${ }_{\Lambda}^{3} \mathrm{H}$ and a very small shift was found in the case of ${ }_{\Lambda}^{4} \mathrm{H}$.

\section{PREDICTIONS FOR FUTURE EXPERIMENTS}

In the following we focus on light hypernuclei and investigate the dependence of cross sections on beam energies as well as on different projectile-target combinations. We restrict this study to carbon projectiles at energies of several $\mathrm{GeV} /$ nucleon and consider carbon and hydrogen targets.

In the top panel of Fig. 5 the production cross section of a ${ }^{12} \mathrm{C}$ beam impinging on a hydrogen target with energies from 1 to $10 \mathrm{GeV} /$ nucleon is shown for ${ }_{\Lambda}^{2,3} n,{ }_{\Lambda}^{3,4,6} \mathrm{H},{ }_{\Lambda}^{7} \mathrm{Li}$, and ${ }_{\Lambda}^{7} \mathrm{Be}$ hypernuclei. The cross sections of all hypernuclei present a steep increase at the production threshold $(1.6 \mathrm{GeV} /$ nucleon) and a linear increase up to $5 \mathrm{GeV} /$ nucleon. A saturation plateau is shown at higher incident energies for all species. The calculation was also performed with a carbon target, and a similar trend of the energy dependence is observed. Such saturation behavior has also been reported in previous calculations $[8,30]$, which is due to the balance between the amount of hyperons and residues with suitable energies such that the capture may happen. In the bottom panel of Fig. 5, we show 


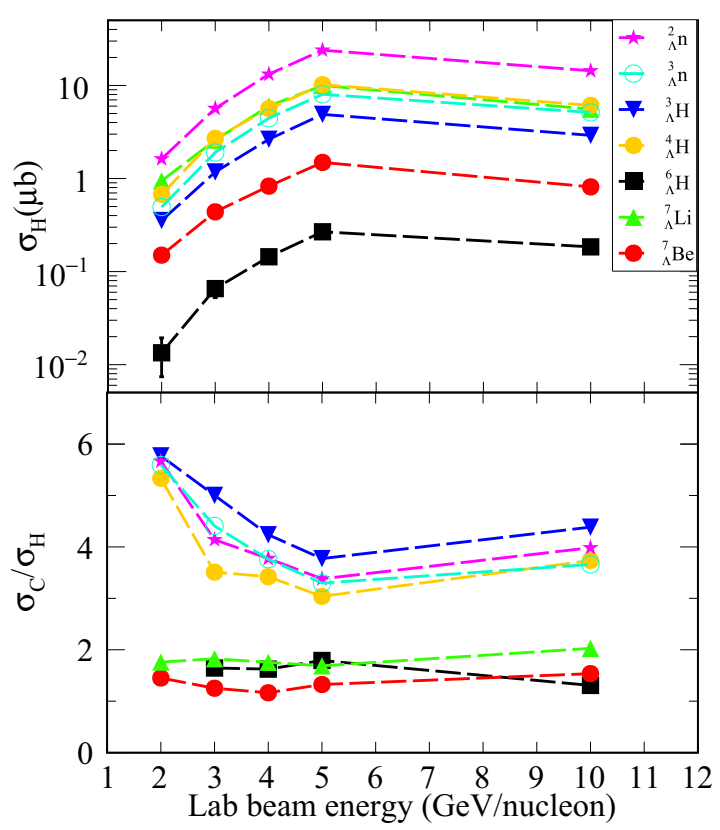

FIG. 5. Top panel: Energy dependence of the hypernuclear production cross section in ${ }^{12} \mathrm{C}+{ }^{1} \mathrm{H}$ collisions at different beam energies. Only the statistical error is considered. Bottom panel: Ratios of hypernuclear production cross sections between ${ }^{12} \mathrm{C}+{ }^{12} \mathrm{C}$ and ${ }^{12} \mathrm{C}+{ }^{1} \mathrm{H}$ collisions at different beam energies. Ratios with large errors are removed. Only projectile-like hypernuclei are considered.

the ratios of hypernuclear production cross sections between ${ }^{12} \mathrm{C}+{ }^{12} \mathrm{C}$ and ${ }^{12} \mathrm{C}+{ }^{1} \mathrm{H}$ collisions at different beam energies. The total inelastic cross sections for a given projectile-target combination do not vary much from 1 to $10 \mathrm{GeV} /$ nucleon. At $2 \mathrm{GeV} /$ nucleon, the inelastic cross section is calculated to be $908 \mathrm{mb}$ for ${ }^{12} \mathrm{C}+{ }^{12} \mathrm{C}$ while it is $268 \mathrm{mb}$ for ${ }^{12} \mathrm{C}+{ }^{1} \mathrm{H}$. At $10 \mathrm{GeV} /$ nucleon, the calculated inelastic cross sections are $892 \mathrm{mb}$ and $271 \mathrm{mb}$, respectively. Therefore, the ratios of the total inelastic cross sections are almost a constant of 3.4 for all of the beam energies. We notice that the crosssection ratios of hypernuclei are separated into two groups by the total inelastic cross-section ratio. For light hypothetical species ${ }_{\Lambda}^{2,3} n$ and ${ }_{\Lambda}^{3,4} \mathrm{H}$, the ratios between hydrogen and carbon targets are roughly equal to the ratios of total inelastic cross sections. With increasing beam energies, there is a general saturation of the excited hyperresidual yields. However, the carbon target favors the production of larger and more excited residues which decay predominantly into small fragments. For large species ${ }_{\Lambda}^{6} \mathrm{H},{ }_{\Lambda}^{7} \mathrm{Li}$, and ${ }_{\Lambda}^{7} \mathrm{Be}$, the production cross sections between carbon and hydrogen targets are comparable through all of the beam energies. The low ratios reflect that the corresponding fraction of low-excited large residues is smaller. In the following discussion, we restrict the incident energies to be $2 \mathrm{GeV} /$ nucleon. Cross sections at higher energies could be evaluated easily from Fig. 5 .

We now investigate the projectile dependence of the production cross sections. The top panel of Fig. 6 shows the production cross sections for ${ }_{\Lambda}^{2,3} n,{ }_{\Lambda}^{3,4,6} \mathrm{H},{ }_{\Lambda}^{7} \mathrm{Li}$, and ${ }_{\Lambda}^{7} \mathrm{Be}$ for ${ }^{10,12,14,16} \mathrm{C}$ beams impinging on a hydrogen target. Intuitively, one could expect that neutron-rich beams may favor the production of

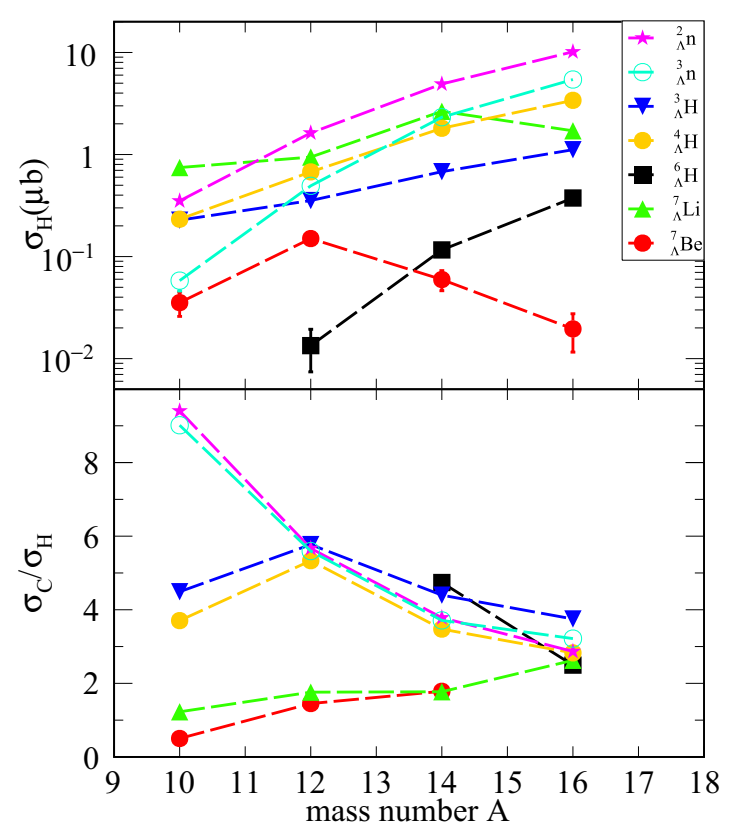

FIG. 6. Top panel: Projectile mass-number dependence of the hypernuclear production cross section in ${ }^{A} \mathrm{C}+{ }^{1} \mathrm{H}$ collisions. Only the statistical error is considered. Bottom panel: Ratios of hypernuclear production cross sections between ${ }^{A} \mathrm{C}+{ }^{12} \mathrm{C}$ and ${ }^{A} \mathrm{C}+{ }^{1} \mathrm{H}$ collisions. Ratios with large errors are removed. The beam energy is fixed at $2 \mathrm{GeV} /$ nucleon and only projectile-like hypernuclei are considered.

neutron-rich hypernuclei. Indeed, as shown in the top panel of Fig. 6, the cross sections of neutron-rich hypernuclei ${ }_{\Lambda}^{2,3} n$ and ${ }_{\Lambda, 4,6} \mathrm{H}$ ) increase as much as two orders of magnitude when the projectile changes from ${ }^{10} \mathrm{C}$ to ${ }^{16} \mathrm{C}$, while the cross sections of proton-rich hypernuclei $\left({ }_{\Lambda}^{7} \mathrm{Be}\right.$ and $\left.{ }_{\Lambda}^{7} \mathrm{Li}\right)$ begin to decrease for ${ }^{16} \mathrm{C}$ projectile. The calculation with a ${ }^{12} \mathrm{C}$ target was also performed, showing similar results. This indicates that the use of high-intensity neutron-rich beams may be an advantage for neutron-rich hypernuclear production. Such measurement can be performed for secondary beam intensity around $10^{5}-10^{7} \mathrm{pps}$ since most of setups are limited by the trigger rates they can handle. If we assume a cross section of $1 \mu \mathrm{b}$ and a 25 -cm-thick hydrogen target, depending on the beam intensity mentioned above, the hypernuclear production rates will be around 0.1-10 pps, which are high enough for a invariant-mass spectroscopy study. The decay of the hypernuclei could happen inside of the target. Given the high beam energy and the small stopping power of the hydrogen target, the use of such a thick hydrogen target does not have large effects on the invariant-mass resolution.

In the bottom panel of Fig. 6 the ratios of production cross sections between a carbon target and a hydrogen target are illustrated. When the projectiles are proton rich, ${ }^{10} \mathrm{C}$ for example, the use of a carbon target results in 5 or 10 times larger cross sections than the use of a hydrogen target in the case of producing light neutron-rich hypernuclei. This is because the yields of such nuclei on the hydrogen target are low, and charge-exchange reactions on the carbon target can increase essentially the neutron content of the projectile residue. As the projectile's mass number increases, the hydrogen target 


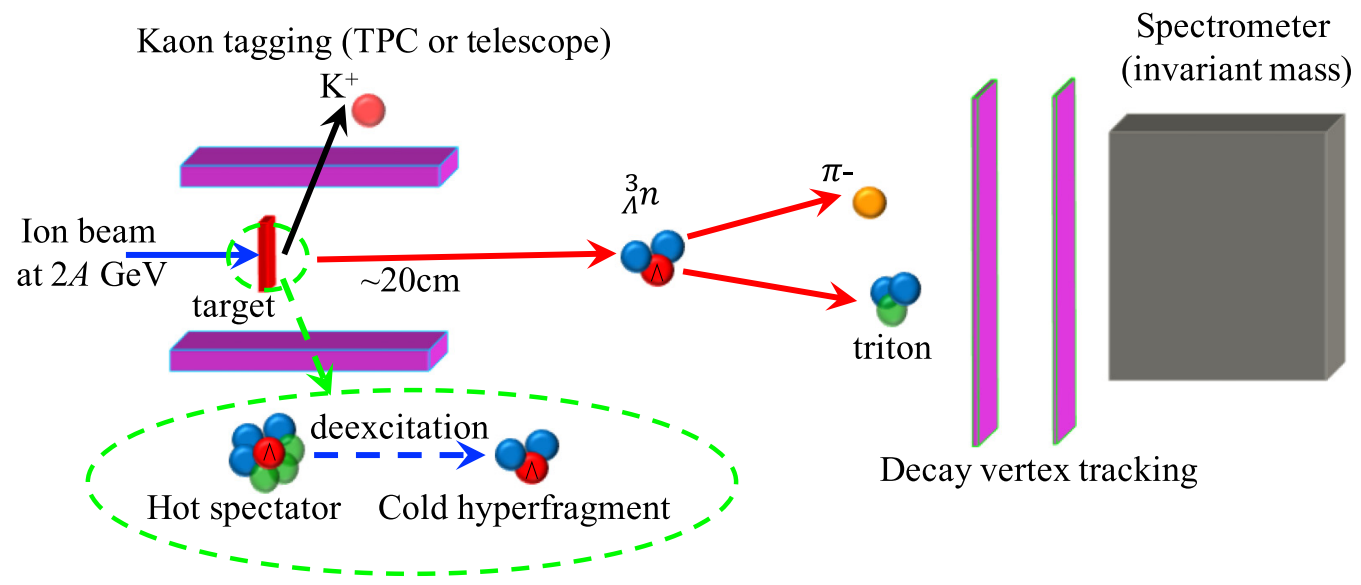

FIG. 7. Schematic diagram of the experimental setup for the invariant-mass spectroscopy of hypernuclei tagged with a kaon.

tends to have production cross sections comparable with the carbon target for any hypernuclei. The gain factor changes from 2 to 4 . Such losses should be easily compensated by using a thicker hydrogen target, because of the much smaller energy loss and smaller inelastic cross sections. The calculated inelastic reaction cross section of ${ }^{12} \mathrm{C}$ on a hydrogen target at $2 \mathrm{~A} \mathrm{GeV}$ is $268 \mathrm{mb}$ while it is $908 \mathrm{mb}$ for a carbon target. With the same beam intensity, a $25-\mathrm{cm}$-thick hydrogen target results in the same luminosity as a 9.5-cm-thick carbon target, while the energy loss in the carbon target is more than 5 times larger.

\section{SIGNAL-OVER-BACKGROUND RATIO}

Hypernuclei can decay through both the mesonic and the nonmesonic weak channels. For light hypernuclei, the mesonic decay mode is favored, in which $\Lambda$ decays to $\pi N$ with a $Q$ value around $40 \mathrm{MeV}$, similar to the decay of a free $\Lambda$. As a consequence of this decay, the $\Lambda$ is substituted by a nucleon and a pion is emitted. The decayed final nucleon has a momentum around $100 \mathrm{MeV} / c$, much less than the Fermi momentum of $280 \mathrm{MeV} / c$. Therefore, in medium-heavy hypernuclei, mesonic decay is suppressed by the Pauli blocking of the nucleonic medium. In parallel, due to a larger overlap of the wave function between $\Lambda$ and nucleons, the nonmesonic decay $\Lambda N \rightarrow N N$ will dominate. For nonmesonic decay, there is no production of $\pi$ and the $Q$ value can be as large as $m_{\Lambda}-m_{N} \approx 176 \mathrm{MeV}$. The final nucleons have enough energy to leave the nucleus or be captured and excite the nucleus. As a result, such excited nucleus can disintegrate into nucleons and multiple heavy fragments. The big challenge of invariant-mass spectroscopy is to clearly identify the production and decay of hypernuclei from their products embedded in a very high background of various particles including pions. It is technically very difficult to measure all the nonmesonic weak decay products of medium-heavy hypernuclei. A nonmesonic decay study of light hypernuclei such as ${ }_{\Lambda}^{4} \mathrm{He} \rightarrow n+n+p+p, d+d$, or $p+t$ should be possible, but the branching ratios are very small [53]. In the present invariant-mass study, we would like to consider the mesonic decay of light hypernuclei. The main contaminants in the hypernuclei identification come from the inelastic reaction channels which could result in production of $\pi$, nucleons, and fragments without forming hypernuclei. The decay of free $\Lambda$ and $\Sigma^{0, \pm}$ is also an important contaminant for the mesonic decay channel. The cross sections of these processes may be an order of magnitude higher than that of the cold hypernuclear production. Intuitively, we expect that a hydrogen target could provide better signal-over-background ratio than an ion target, since the reduced number of nucleons will reduce the amount of produced pions and an exclusive measurement will be in principle easier to reach. To investigate the signal-over-background ratio quantitatively for both carbon and hydrogen targets, we perform a simulation using the theoretical events as an input. After Fermi breakup, the produced cold hypernuclei undergo mesonic or nonmesonic decay with a lifetime of hundreds of picoseconds. Decay of $\Lambda$ and $\Sigma^{0, \pm}$ was also considered. Due to the inverse kinematic and limitation of the acceptance, here we only focus on the projectile-like hypernuclei. Target-like particles were removed from the simulation since they can be easily rejected by a proper momentum acceptance setting in the experiment. Only particles with rapidity larger than zero and moving in very forward angle $\left(\theta_{\text {lab }}<10^{\circ}\right)$ were considered as accepted. The reconstructed hypernuclei were required to have rapidity of $y>0.75 y_{\text {proj }}$ and the scattering angles were required to be less than $5^{\circ}$, where $y_{\text {proj }}$ is the rapidity of the projectile in the laboratory frame. In our simulation, a constant momentum resolution $\left(\sigma_{p} / p=1 \%\right)$ was taken for all kinetic energies of $\pi$, proton, neutron and heavy fragments. This leads to a resolution (FWHM) of $2.5 \mathrm{MeV}$ for $\Lambda$ ( $\pi^{-}+$proton). In addition, $5 \mathrm{~mm}(1 \sigma)$ spatial resolution was considered for the production and decay vertices in $x, y$, and $z$ directions. To reduce the huge background, we require only events with strangeness production, which coincides with kaon production around the target in the experiment. The lifetime of the $K^{+}$ meson is $12 \mathrm{~ns}$ and it will decay to $\mu^{+}+v_{\mu}$ or $\pi^{+}+\pi^{0}$ with branching ratios of $63.5 \%$ and $21.2 \%$ respectively. It has been shown that $K^{+}$can be efficiently identified either in flight with a time projection chamber (TPC) [54] or at rest using a kaon range telescope [55]. A schematic diagram of the experimental setup is shown in Fig. 7.

Recently, exotic bound hypernuclei, like ${ }_{\Lambda}^{2} n$ and ${ }_{\Lambda}^{3} n$, were extensively discussed and looked for in relativistic ion 
experiments [22,56]. As examples, we consider here the mesonic decay processes of ${ }_{\Lambda}^{2} n$ and ${ }_{\Lambda}^{3} n$, i.e., ${ }_{\Lambda}^{2} n \rightarrow \pi^{-}+d$ and ${ }_{\Lambda}^{3} n \rightarrow \pi^{-}+t$. Since the lifetimes of ${ }_{\Lambda}^{2} n$ and ${ }_{\Lambda}^{3} n$ are still unknown, the possible lifetimes of 181 and 190 ps were used in the simulation for ${ }_{\Lambda}^{2} n$ and ${ }_{\Lambda}^{3} n$ [22]. Invariant masses of ${ }_{\Lambda}^{2} n$ and ${ }_{\Lambda}^{3} n$ were reconstructed from the momenta of $\pi^{-}+d$ and $\pi^{-}+$ $t$, respectively. The obtained invariant-mass spectra are shown in Fig. 8 for ${ }^{12} \mathrm{C}$ beams impinging on carbon and hydrogen targets at $2 \mathrm{~A} \mathrm{GeV}$. We note that all of the combinations were considered in the invariant-mass reconstruction if there were multiple $\pi^{-}, d$, and $t$ accepted. For direct comparison, the number of collision events, the experimental acceptance, as well as the reconstruction of hypernuclei were the same for each plot. From Fig. 8, we can clearly see the improvement of the signal-over-background ratio when using a hydrogen target. We found that one main reason is the reduction of $\pi^{-}$ background at forward angles. In the ${ }^{12} \mathrm{C}+{ }^{12} \mathrm{C}$ collisions, $78 \%$ of the $\pi^{-}$background comes from the cascade collisions, while in the case of ${ }^{12} \mathrm{C}+$ proton, this ratio drops to $27 \%$. After coincidence with kaons, most of the $\pi^{-}$background in ${ }^{12} \mathrm{C}+$ proton collisions come from the decay of free lambda particles, which is the main background for the mesonic decay channels. We note that vertex methods for identification of products of slow weak decays have been successfully achieved in several hypernuclear experiments [18,21,22]. From the simulation results in Ref. [57], the $\pi^{-}$background can be reduced to $1.7 \%$ by applying a vertex trigger in the ${ }^{6} \mathrm{Li}$ and ${ }^{12} \mathrm{C}$ collisions at $2 \mathrm{~A} \mathrm{GeV}$. The background suppression can be clearly seen in the bottom panel of Fig. 8, where the distance between the production and decay vertices $(\Delta R)$ was required to be larger than $1.5 \mathrm{~cm}$, which is about two times the resolution of the distance. In our simulation, multiple scattering of pions and light ions in the target was not considered, so we expect a worse signal-over-background ratio especially for the carbon target. Thus, we foresee in a future work further study of the performance of a realistic setup dedicated to hypernuclei production from hydrogen induced reactions.

\section{SUMMARY}

Ion beam induced reactions are a very promising way to produce exotic hypernuclei, as already proved by the HypHI collaboration at GSI. In this article, we present a series of calculations using the Dubna intranuclear cascade model followed by Fermi breakup to investigate theoretically the production of light $\Lambda$ hypernuclei. The calculated cross sections are compared with available experimental data. We found that the Dubna data could be fairly well reproduced if we slightly tune the excitation-energy distribution of the hot primary hyperresidues. However, the calculated yields of hypernuclei are more than one order of magnitude smaller than the recently published HypHI data. With a more detailed comparison of rapidity and transverse momentum distributions, we confirm that the observed hypernuclei in the HypHI experiment are mainly projectile-like hypernuclei with a small cascadecoalescence contribution. Although the amplitudes are much smaller and there exists some rapidity shift, the overall shape of both rapidity and transverse momentum distributions agree with the published data. Furthermore, we also investigate
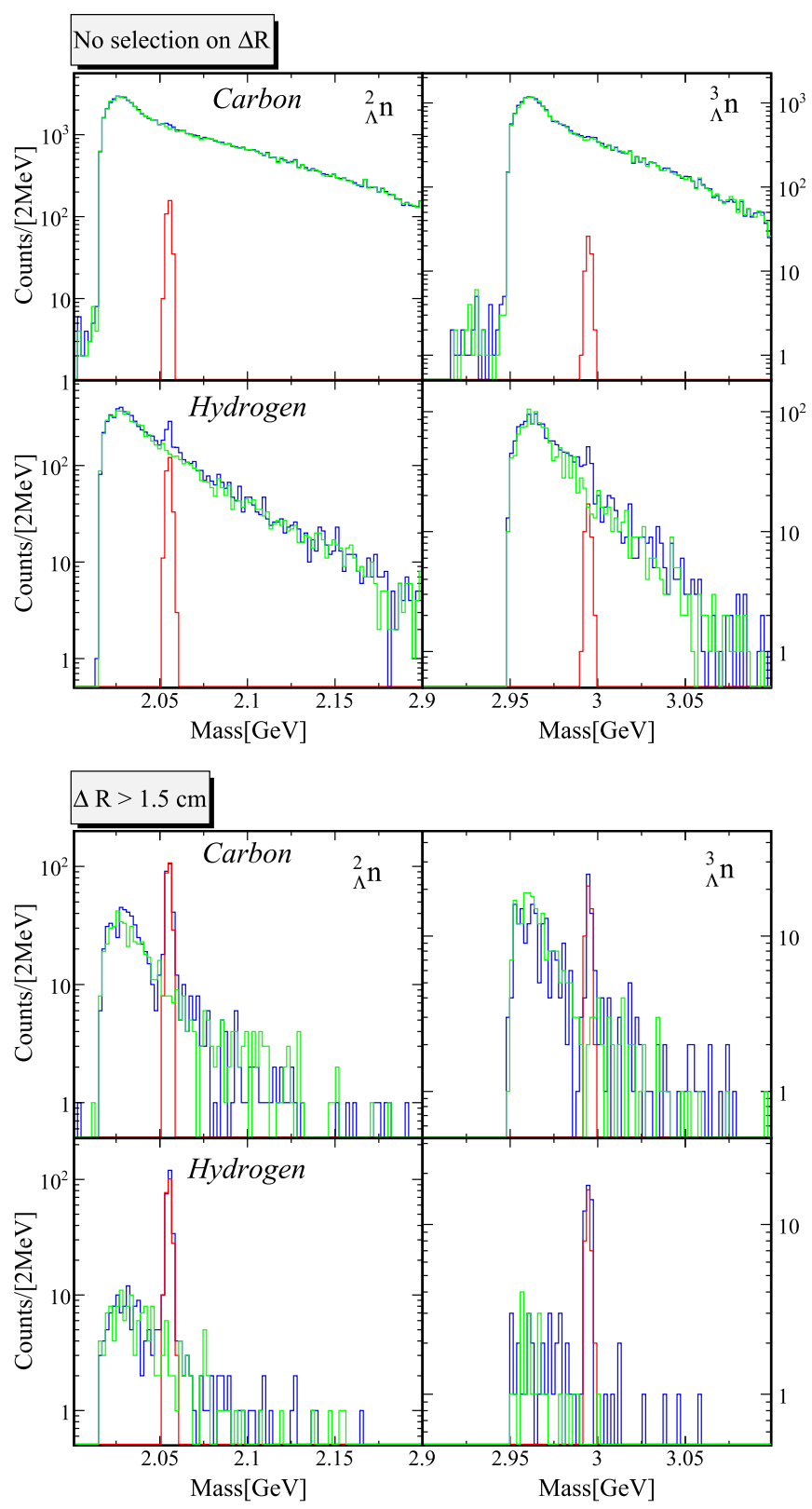

FIG. 8. Invariant-mass spectrums of ${ }_{\Lambda}^{2} n$ and ${ }_{\Lambda}^{3} n$ using a ${ }^{12} \mathrm{C}$ beam at $2 \mathrm{GeV} /$ nucleon impinging on a carbon target and a hydrogen target. $\Delta R$ denotes the distance between production and decay vertices. For the figures in the top panel, there is no selection on $\Delta R$ in the invariant-mass reconstruction. For the figures in the bottom panel, $\Delta R$ is selected to be larger than $1.5 \mathrm{~cm}$. The red spectra show the invariant mass of ${ }_{\Lambda}^{2} n$ or ${ }_{\Lambda}^{3} n$ obtained using only the corresponding decay particles. The green spectra show the background contaminations obtained using uncorrelated particles. The blue spectra are obtained if we consider both uncorrelated particles and decay particles in the invariant-mass reconstruction.

the cross-section dependence on beam energies and different projectile-target combinations. Comparing with carbon target, hydrogen target also leads to sizable hypernuclear yields, even for exotic species. In the presented calculations, the cross-section ratios between carbon and hydrogen targets 
are similar to the total inelastic cross-section ratios, making hydrogen a competitive target for hypernuclear production in relativistic ion collisions. The typical hypernuclear production cross sections at $2 \mathrm{~A} \mathrm{GeV}$ beam energy with a hydrogen target are around $0.5 \mu \mathrm{b}$. From the experimental point of view, we also investigate the signal-over-background ratio using a ${ }^{12} \mathrm{C}$ beam impinging on hydrogen and carbon targets. Invariantmass spectra of ${ }_{\Lambda}^{2} n$ and ${ }_{\Lambda}^{3} n$ are given, taking into account the experimental acceptance and resolution. With these examples, we demonstrate that a hydrogen target could indeed reduce significantly the background contamination in the mesonic decay channel for some experiments. Hypernuclear production data from ion collisions with hydrogen and carbon targets are required to benchmark the current predictions and allow for the development of future experimental programs at the FAIR facility in GSI and the HIAF facility in China.

\section{ACKNOWLEDGMENTS}

Y.L.S. acknowledges the support of a Marie SkłodowskaCurie Individual Fellowship (H2020-MSCA-IF-2015-705023) from the European Union. A.S.B. acknowledges the support of BMBF (Germany). The authors thank K. K. Gudima for clarifying discussions of the calculations and J. Pochodzalla for stimulating this work. Valuable discussions with C. Rappold, T. Saito, and C. Scheidenberger are gratefully acknowledged. Support from ESNT for organizing the hypernuclear workshop at CEA is gratefully acknowledged.
[1] M. Danysz and J. Pniewski, London Edinburgh Dublin Philos. Mag. J. Sci. 44, 348 (1953).

[2] C. Amsler et al. (Particle Data Group), Phys. Lett. B 667, 1 (2008).

[3] A. Gal, E. V. Hungerford, and D. J. Millener, Rev. Mod. Phys. 88, 035004 (2016).

[4] H. Hotchi, T. Nagae, H. Outa, H. Noumi, M. Sekimoto, T. Fukuda, H. Bhang, Y. D. Kim, J. H. Kim, H. Park, K. Tanida, O. Hashimoto, H. Tamura, T. Takahashi, Y. Sato, T. Endo, S. Satoh, J. Nishida, T. Miyoshi, T. Saitoh, T. Kishimoto, A. Sakaguchi, S. Ajimura, Y. Shimizu, T. Mori, S. Minami, M. Sumihama, R. Sawafta, and L. Tang, Phys. Rev. C 64, 044302 (2001).

[5] K. Tanida, H. Tamura, D. Abe, H. Akikawa, K. Araki, H. Bhang, T. Endo, Y. Fujii, T. Fukuda, O. Hashimoto, K. Imai, H. Hotchi, Y. Kakiguchi, J. H. Kim, Y. D. Kim, T. Miyoshi, T. Murakami, T. Nagae, H. Noumi, H. Outa, K. Ozawa, T. Saito, J. Sasao, Y. Sato, S. Satoh, R. I. Sawafta, M. Sekimoto, T. Takahashi, L. Tang, H. H. Xia, S. H. Zhou, and L. H. Zhu, Phys. Rev. Lett. 86, 1982 (2001).

[6] K. Hagino, J. Yao, F. Minato, Z. Li, and M. T. Win, Nucl. Phys. A 914, 151 (2013).

[7] C. Samanta, P. R. Chowdhury, and D. N. Basu, J. Phys. G: Nucl. Part. Phys. 35, 065101 (2008).

[8] A. S. Botvina, K. K. Gudima, and J. Pochodzalla, Phys. Rev. C 88, 054605 (2013).

[9] M. Agnello et al. (FINUDA Collaboration), Phys. Rev. Lett. 108, 042501 (2012).

[10] M. Jurić, G. Bohm, J. Klabuhn, U. Krecker, G. CoremansBertrand, J. Sacton, T. Cantwell, F. Esmael, A. Montwill, D. Davis, D. Kiełczewska, T. Tymieniecka, and J. Zakrzewski, Nucl. Phys. B 35, 160 (1971).

[11] J. L. Zdunik and P. Haensel, Astron. Astrophys. 551, A61 (2013).

[12] D. Lonardoni, A. Lovato, S. Gandolfi, and F. Pederiva, Phys. Rev. Lett. 114, 092301 (2015).

[13] S. N. Nakamura et al. [HKS (JLab E01-011) Collaboration], Phys. Rev. Lett. 110, 012502 (2013).

[14] K. J. Nield, T. Bowen, G. D. Cable, D. A. DeLise, E. W. Jenkins, R. M. Kalbach, R. C. Noggle, and A. E. Pifer, Phys. Rev. C 13, 1263 (1976).

[15] S. Avramenko, V. Aksinenko, M. Anikina, B. Bannik, Y. Belikov, V. Butenko, V. Drozdov, K. Gajevski, A. Galperin, N. Glagoleva, A. Golokhvastov, K. Iovchev, N. Kaminsky, S. Khorozov, E.
Khusainov, V. Kondratiev, E. Kozubsky, L. Krasnov, B. Kulakov, J. Lukstin, O. Mandrik, A. Matyushin, V. Matyushin, J. Mirkovski, S. Mukhin, N. Nikityuk, N. Nurgozhin, L. Okhrimenko, E. Okonov, T. Ostanevich, Z. Pavlovski, A. Piatkovski, Y. Pol, V. Radomanov, I. Saitov, S. Sedikh, I. Shevchenko, I. Stepanov, G. Taran, G. Vardenga, and V. Zavyalov, Nucl. Phys. A 547, 95 (1992).

[16] T. A. Armstrong et al. (E864 Collaboration), Phys. Rev. C 70, 024902 (2004).

[17] B. I. Abelev et al. (The STAR Collaboration), Science 328, 58 (2010).

[18] B. Dönigus, Nucl. Phys. A 904-905, 547c (2013).

[19] A. S. Botvina, J. Steinheimer, and M. Bleicher, Phys. Rev. C 96, 014913 (2017).

[20] A. Botvina, J. Steinheimer, E. Bratkovskaya, M. Bleicher, and J. Pochodzalla, Phys. Lett. B 742, 7 (2015).

[21] C. Rappold, E. Kim, D. Nakajima, T. Saito, O. Bertini, S. Bianchin, V. Bozkurt, M. Kavatsyuk, Y. Ma, F. Maas, S. Minami, B. Özel-Tashenov, K. Yoshida, P. Achenbach, S. Ajimura, T. Aumann, C. A. Gayoso, H. Bhang, C. Caesar, S. Erturk, T. Fukuda, B. Göküzüm, E. Guliev, T. Hiraiwa, J. Hoffmann, G. Ickert, Z. Ketenci, D. Khaneft, M. Kim, S. Kim, K. Koch, N. Kurz, A. L. Fèvre, Y. Mizoi, M. Moritsu, T. Nagae, L. Nungesser, A. Okamura, W. Ott, J. Pochodzalla, A. Sakaguchi, M. Sako, C. Schmidt, M. Sekimoto, H. Simon, H. Sugimura, T. Takahashi, G. Tambave, H. Tamura, W. Trautmann, S. Voltz, N. Yokota, and C. Yoon, Nucl. Phys. A 913, 170 (2013).

[22] C. Rappold et al. (HypHI Collaboration), Phys. Rev. C 88, 041001(R) (2013).

[23] H. H. Gutbord, I. Augustin, H. Eickhoff, K.-D. Groß, W. F. Henning, D. Krämer, G. Walter, FAIR Baseline Technical Report, Tech. Rep. (GSI, Darmstadt, 2006).

[24] J. Äystö, K.-H. Behr, J. Benlliure, A. Bracco, P. Egelhof, A. Fomichev, S. Galès, H. Geissel, T. Grahn, L. Grigorenko, M. Harakeh, R. Hayano, S. Heinz, K. Itahashi, A. Jokinen, N. Kalantar-Nayestanaki, R. Kanungo, H. Lenske, I. Mukha, G. Münzenberg, C. Nociforo, H. Ong, S. Pietri, M. Pfützner, W. Plaß, A. Prochazka, S. Purushothaman, T. Saito, C. Scheidenberger, H. Simon, I. Tanihata, S. Terashima, H. Toki, L. Trache, H. Weick, J. Winfield, M. Winkler, and V. Zamfir, Nucl. Instrum. Methods Phys. Res., Sect. B 376, 111 (2016).

[25] J. Yang, J. Xia, G. Xiao, H. Xu, H. Zhao, X. Zhou, X. Ma, Y. He, L. Ma, D. Gao, J. Meng, Z. Xu, R. Mao, W. Zhang, Y. Wang, L. 
Sun, Y. Yuan, P. Yuan, W. Zhan, J. Shi, W. Chai, D. Yin, P. Li, J. Li, L. Mao, J. Zhang, and L. Sheng, Nucl. Instrum. Methods Phys. Res., Sect. B 317, 263 (2013).

[26] C. Rappold and J. López-Fidalgo, Phys. Rev. C 94, 044616 (2016).

[27] R. Ogul, A. S. Botvina, U. Atav, N. Buyukcizmeci, I. N. Mishustin, P. Adrich, T. Aumann, C. O. Bacri, T. Barczyk, R. Bassini, S. Bianchin, C. Boiano, A. Boudard, J. Brzychczyk, A. Chbihi, J. Cibor, B. Czech, M. De Napoli, J.-E. Ducret, H. Emling, J. D. Frankland, M. Hellström, D. Henzlova, G. Immè, I. Iori, H. Johansson, K. Kezzar, A. Lafriakh, A. Le Fèvre, E. Le Gentil, Y. Leifels, J. Lühning, J. Łukasik, W. G. Lynch, U. Lynen, Z. Majka, M. Mocko, W. F. J. Müller, A. Mykulyak, H. Orth, A. N. Otte, R. Palit, P. Pawłowski, A. Pullia, G. Raciti, E. Rapisarda, H. Sann, C. Schwarz, C. Sfienti, H. Simon, K. Sümmerer, W. Trautmann, M. B. Tsang, G. Verde, C. Volant, M. Wallace, H. Weick, J. Wiechula, A. Wieloch, and B. Zwiegliński, Phys. Rev. C 83, 024608 (2011).

[28] J. Bondorf, A. Botvina, A. Iljinov, I. Mishustin, and K. Sneppen, Phys. Rep. 257, 133 (1995).

[29] A. S. Botvina, K. K. Gudima, J. Steinheimer, M. Bleicher, and I. N. Mishustin, Phys. Rev. C 84, 064904 (2011).

[30] A. S. Botvina, K. K. Gudima, J. Steinheimer, M. Bleicher, and J. Pochodzalla, Phys. Rev. C 95, 014902 (2017).

[31] V. Toneev, N. Amelin, K. Gudima, and S. Sivoklokov, Nucl. Phys. A 519, 463 (1990).

[32] T. Sjöstrand, S. Ask, J. R. Christiansen, R. Corke, N. Desai, P. Ilten, S. Mrenna, S. Prestel, C. O. Rasmussen, and P. Z. Skands, Comput. Phys. Commun. 191, 159 (2015).

[33] B. Nilsson-Almqvist and E. Stenlund, Comput. Phys. Commun. 43, 387 (1987).

[34] V. Toneev and K. Gudima, Nucl. Phys. A 400, 173 (1983).

[35] Z. Fraenkel, Nucl. Phys. A 428, 373 (1984).

[36] S. Bass, M. Belkacem, M. Bleicher, M. Brandstetter, L. Bravina, C. Ernst, L. Gerland, M. Hofmann, S. Hofmann, J. Konopka, G. Mao, L. Neise, S. Soff, C. Spieles, H. Weber, L. Winckelmann, H. Stöcker, W. Greiner, C. Hartnack, J. Aichelin, and N. Amelin, Prog. Part. Nucl. Phys. 41, 255 (1998).

[37] M. Bleicher, E. Zabrodin, C. Spieles, S. A. Bass, C. Ernst, S. Soff, L. Bravina, M. Belkacem, H. Weber, H. Stöcker, and W. Greiner, J. Phys. G: Nucl. Part. Phys. 25, 1859 (1999).

[38] O. Buss, T. Gaitanos, K. Gallmeister, H. van Hees, M. Kaskulov, O. Lalakulich, A. Larionov, T. Leitner, J. Weil, and U. Mosel, Phys. Rep. 512, 1 (2012).

[39] W. Cassing and E. L. Bratkovskaya, Phys. Rev. C 78, 034919 (2008).

[40] T. Gaitanos, H. Lenske, and U. Mosel, Phys. Lett. B 675, 297 (2009).

[41] M. Wakai, H. Bando, and M. Sano, Phys. Rev. C 38, 748 (1988).

[42] J. Steinheimer, K. Gudima, A. Botvina, I. Mishustin, M. Bleicher, and H. Stöcker, Phys. Lett. B 714, 85 (2012).
[43] A. S. Botvina, M. Bleicher, J. Pochodzalla, and J. Steinheimer, Eur. Phys. J. A 52, 242 (2016).

[44] A. Botvina and I. Mishustin, Phys. Lett. B 294, 23 (1992).

[45] S. Hudan et al. (INDRA Collaboration), Phys. Rev. C 67, 064613 (2003).

[46] A. S. Botvina and J. Pochodzalla, Phys. Rev. C 76, 024909 (2007).

[47] A. S. Lorente, A. S. Botvina, and J. Pochodzalla, Phys. Lett. B 697, 222 (2011).

[48] A. S. Botvina, N. Buyukcizmeci, A. Ergun, R. Ogul, M. Bleicher, and J. Pochodzalla, Phys. Rev. C 94, 054615 (2016).

[49] A. Botvina, I. Mishustin, M. Begemann-Blaich, J. Hubele, G. Imme, I. Iori, P. Kreutz, G. Kunde, W. Kunze, V. Lindenstruth, U. Lynen, A. Moroni, W. Müller, C. Ogilvie, J. Pochodzalla, G. Raciti, T. Rubehn, H. Sann, A. Schüttauf, W. Seidel, W. Trautmann, and A. Wörner, Nucl. Phys. A 584, 737 (1995).

[50] C. Rappold (private communication).

[51] C. Rappold, T. Saito, O. Bertini, S. Bianchin, V. Bozkurt, E. Kim, M. Kavatsyuk, Y. Ma, F. Maas, S. Minami, D. Nakajima, B. Özel-Tashenov, K. Yoshida, P. Achenbach, S. Ajimura, T. Aumann, C. A. Gayoso, H. Bhang, C. Caesar, S. Erturk, T. Fukuda, B. Göküzüm, E. Guliev, J. Hoffmann, G. Ickert, Z. Ketenci, D. Khaneft, M. Kim, S. Kim, K. Koch, N. Kurz, A. L. Fèvre, Y. Mizoi, L. Nungesser, W. Ott, J. Pochodzalla, A. Sakaguchi, C. Schmidt, M. Sekimoto, H. Simon, T. Takahashi, G. Tambave, H. Tamura, W. Trautmann, S. Voltz, and C. Yoon, Phys. Lett. B 747, 129 (2015).

[52] A. Le Fèvre, Y. Leifels, J. Aichelin, C. Hartnack, V. Kireyev, and E. Bratkovskaya, J. Phys.: Conf. Ser. 668, 012021 (2016).

[53] M. Agnello, L. Benussi, M. Bertani, H. Bhang, G. Bonomi, E. Botta, M. Bregant, T. Bressani, S. Bufalino, L. Busso, D. Calvo, P. Camerini, B. Dalena, F. D. Mori, G. D’Erasmo, F. Fabbri, A. Feliciello, A. Filippi, E. Fiore, A. Fontana, H. Fujioka, P. Genova, P. Gianotti, N. Grion, B. Kang, V. Lucherini, S. Marcello, T. Maruta, N. Mirfakhrai, F. Moia, O. Morra, T. Nagae, D. Nakajima, H. Outa, A. Pantaleo, V. Paticchio, S. Piano, F. Pompili, R. Rui, G. Simonetti, A. Toyoda, R. Wheadon, and A. Zenoni, Nucl. Phys. A 835, 439 (2010).

[54] J. Adam et al. (ALICE Collaboration), Eur. Phys. J. C 75, 226 (2015)

[55] T. Armstrong, D. Elias, R. Lewis, E. Minor, J. Passaneau, and G. Smith, Nucl. Instrum. Methods Phys. Res., Sect. A 289, 109 (1990).

[56] B. Dönigus, EPJ Web Conf. 97, 00013 (2015).

[57] S. Minami, P. Achenbach, S. Ajimura, S. Bianchin, O. Borodina, T. Fukuda, Y. Hayashi, T. Hiraiwa, J. Hoffmann, M. Kavatsyuk, N. Kurz, O. Lepyoshkina, Y. Mizoi, T. Mochizuki, M. Moritsu, T. Nagae, D. Nakajima, W. Ott, J. Pochodzalla, C. Rappold, A. Sakaguchi, T.R.Saito, K. Tanida, and W. Trautmann, GSI Scientific Report 2007 (GSI, Darmstadt, 2008), p. 223, https://repository.gsi.de/record/53524/files/. 